\title{
Voluntary allocation versus automatic capture of visual attention
}

\author{
C. BRUCE WARNER, JAMES F. JUOLA, and HIDEYA KOSHINO \\ University of Kansas, Lawrence, Kansas
}

\begin{abstract}
Is there a difference in the kind of attention elicited by an abrupt-onset peripheral cue and that elicited by an instruction (e.g., a central arrow cue) to move attention to a peripheral location? In Experiment 1, we found that peripheral cues are no more effective in orienting attention than are central cues. No evidence was found for separable attentional systems consisting of a volitional response to central cues and an automatic response triggered only by peripheral cues. Rather, an identical or similar attentional process seems to be activated by either type of cue, although perhaps in different ways. Peripheral cues seem to have an automatic component, however, in that once attention is engaged by a peripheral cue, it cannot easily be disengaged for refocus elsewhere. In Experiment 2, after several sessions of practice, subjects were able to circumvent automatic attentional capture by an abrupt-onset peripheral cue and to volitionally redirect the focus of attention. Thus, attentional capture by abrupt-onset stimuli is not strongly automatic.
\end{abstract}

Selective visual attention is often likened to a spotlight that can be oriented at will, or to a zoom lens that can be expanded or contracted as necessary to enhance processing in contiguous spatial locations (Eriksen \& St. James, 1986; Eriksen \& Yeh, 1985; LaBerge, 1983). Posner (1980; Posner \& Cohen, 1984) proposed two sources of control for the attentional spotlight: (1) endogenous or volitional orienting in response to a symbolic indicator (such as a central cue), and (2) exogenous or nonvolitional orienting to a source of extrafoveal stimulation (such as a peripheral cue).

Jonides (1981) directly compared the effects of peripheral and central cues. In his task, either a central arrow at the fixation point or a peripheral arrow near one of eight positions in a circular array around the fixation point was given in advance of a display. The display contained seven distractor letters and one critical letter $(R$ or L). The response times (RTs), indicating which critical letter was present, showed benefits for valid position cues and costs for invalid cues relative to the times obtained on neutral, no-cue trials. Jonides found four important differences between the effects of central and peripheral cues, in that peripheral cues: (1) do not utilize processing resources as heavily, (2) produce attentional responses that are more difficult to suppress (even

The research reported here was presented at meetings of the Psychonomic Society in Chicago (1988) and Atlanta (1989). Support for this research was provided by grants to the second author from the General Research Fund and Biomedical Sciences Committees of the University of Kansas. Experiment 1 is based on a master's thesis submitted by the first author to the University of Kansas. We acknowledge the assistance of Dwight O'Neal and Preston Pratt in conducting Experiment 2. Thanks are also due Steven Yantis and Lester Krueger for helpful comments on an earlier draft of this manuscript. Reprint requests should be addressed to James F. Juola, Psychology Department, 426 Fraser Hall, University of Kansas, Lawrence, KS 66045. when subjects are instructed to ignore them), (3) are more effective in summoning attention (they produce greater costs and benefits), and (4) are effective even when their onsets are unexpected. Peripheral cues might exert control over attention in a special way, perhaps through transient visual channels excited by abrupt onset, motion, or flicker (Todd \& Van Gelder, 1979; Yantis \& Jonides, 1984). In this way, a single attentional mechanism could be activated either automatically, through a transientdetection system, or volitionally in response to instructions.

Müller and Rabbitt (1989) raised the alternative possibility that there might be qualitative differences between the types of attention elicited volitionally and nonvolitionally. They presented four boxes centered around a fixation point in which either a target, " $\mathrm{T}$," or a distractor, " + ," appeared. Their central cue was an arrow at the fixation point pointing at one of the boxes; their peripheral cue was a brief increase in the brightness of one of the boxes itself. In their first experiment, peripheral cues produced large costs and benefits in target identification. Costs plus benefits grew rapidly and reached peak magnitudes at cue-target stimulus onset asynchronies (SOAs) in the range of $100-150 \mathrm{msec}$. Central cues produced smaller costs and benefits with a longer rise time, reaching a peak between 200 - and 400 -msec SOA, after which the costs and benefits were the same for both types of cues. Their second and third experiments indicated that it was difficult for subjects to override nonvolitional orientation, at least during the first $400 \mathrm{msec}$ after the onset of a peripheral cue. The effects of peripheral cues were also found to be more resistant to the influence of competing flashes than were the effects of central cues, again suggesting that they capture attention automatically. Müller and Rabbitt concluded that peripheral cues activate a more primitive orienting mechanism than do central cues, a 
mechanism that is characterized by transient channel excitation and automatic mobilization of resources.

Briand and Klein (1987) also supported a distinction between the properties of volitional and nonvolitional orienting. They found that peripheral cues elicited the featureconjoining attention hypothesized by feature integration theory (FIT; Treisman \& Gelade, 1980), whereas central cues did not. Two of four letters (a target, R, and distractors, $\mathrm{P}, \mathrm{B}$, or $\mathrm{Q}$ ) were presented to the left or right of fixation (a pair of slash-mark patches was placed on the opposite side to prevent attention from being drawn reflexively to the letters). An arrow precue validly directed subjects to the letter pair on $80 \%$ of the trials. The relevant data came from target-absent trials in which the pair of distractor letters either could (PQ) or could not (PB) give rise to the false detection of an " $R$ ' by illusory conjunction. If the attention evoked by any cue accomplishes the feature integration envisaged by FIT, then costs and benefits should be greater when an illusory conjunction is possible (the PQ distractors). The predicted difference in costs and benefits was realized only with peripheral cues, leading Briand and Klein to conclude that the attention oriented in response to peripheral cues might not be the same as that oriented in response to central cues.

In summary, there are at least three related views of how peripheral and central cues differentially affect visual attention: (1) Both types of cues work in similar ways on a common attentional mechanism, with peripheral cues perhaps acting more strongly through both automatic and volitional components, whereas central cues exert volitional control only (e.g., Jonides \& Yantis, 1988; Yantis \& Jonides, 1984). (2) Peripheral cues activate a separate central mechanism, which follows transient channels in the visual system to activate attention more rapidly and powerfully than central cues. Since there is only a single attention system, the automatic control of peripheral cues usually dominates any competing voluntary controls (e.g., Müller \& Rabbitt, 1989). (3) The attention system activated automatically by peripheral cues is separate from the one activated voluntarily in response to central cues (e.g., Briand \& Klein, 1987).

The separable systems hypothesis asserts that the attentional resources mobilized automatically are independent of those mobilized through effortful processing. If this hypothesis is true, then the simultaneous use of both cue types should result in two simultaneous attentional responses, with separate costs and benefits observable for each.

In Experiment 1, we tested the separable systems hypothesis by using a peripheral cue simultaneously as a high-validity volitional cue and a low-validity nonvclitional cue. Subjects were instructed to attend to the position diametrically opposite a peripheral cue arrow (see Eriksen \& Collins, 1969). If the separable systems hypothesis is correct, a benefit should be observed both for targets appearing opposite the peripheral cue (because of the controlled allocation of attention) and for targets appearing near the peripheral cue (because of automatic capture of attention by the onset of the arrow). Costs would be observed for targets appearing at positions irrelevant for either interpretation of the cue arrow. If, on the other hand, a single attentional system is activated, there should be tradeoffs varying over time between the amount of benefits shown for the peripherally and centrally cued positions.

In Experiment 1, we also tested Jonides's (1981) assertion that, because of the automatic orientation of attention to the source of abrupt peripheral or parafoveal stimulation or to the different properties of nonvolitional and volitional attention, peripheral cues are more efficient at drawing attention than are comparable central cues. If a peripheral cue acts automatically by summoning attention to its spatial location, then one would expect its effects to appear sooner than a central cue's effects (e.g., Müller \& Rabbitt, 1989).

Experiment 2 was an extended replication of part of Experiment 1 . It was designed to determine whether subjects could learn to disengage the attention-capturing property of peripheral cues and refocus attention rapidly on another spatial position. Subjects received consistent training over several sessions, enabling us to demonstrate that extended practice can lead not only to automatization of controlled processes (e.g., Schneider \& Shiffrin, 1977; Shiffrin \& Schneider, 1977), but also to the voluntary control of an automatic process (as suggested by Jonides, 1981).

\section{EXPERIMENT 1}

To test whether the cost and benefit effects of peripheral cues are larger and appear sooner than those of central cues, a variation on a fairly standard spatial priming paradigm was employed. Separate groups of subjects were presented with spatial precues that pointed to one of four potential target positions. These cues were valid, invalid, or neutral indicators of the target (all four positions were indicated in the neutral condition in order to measure both costs of invalid cues and benefits of valid cues). One group received only central cues; the other, only peripheral cues, and there were four cue-display SOAs $(0,50,100$, or $150 \mathrm{msec}$ ).

To determine whether peripheral cues automatically draw attention to their spatial locus, a third group of subjects also received peripheral precues, but, instead of directly indicating a nearby character, the cue arrow signaled subjects to attend to the diametrically opposed display position. Thus the peripheral cue acted as a volitional cue by requiring interpretation and voluntary allocation of attention to a distant location. Two properties, taken together, are generally deemed sufficient to distinguish an automatic process from a controlled (or at least a tenuously automatic) process (LaBerge, 1981): First, automatic processes are insensitive to a concurrent cognitive or perceptual load. Second, they cannot be suppressed or interrupted once they are activated. In a weaker version of this second property, automatic processes can be suppressed in some ways and at some time after being initiated. Assuming that this weaker assumption holds true 
for a single attentional system, benefits are expected to accrue initially to the position near the onset of the peripheral cue and later switch to the diametrically opposed position as attentional resources are disengaged and volitionally reallocated. Relative to the Central group, then, the appearance of benefits should be delayed. Conversely, costs should appear initially for the opposite display position, followed later by the appearance of costs at the position near the peripheral cue.

The use of a peripheral cue as a volitional cue should also reveal whether central and peripheral cues elicit two separate attentional systems (Briand \& Klein, 1987). For the separable systems hypothesis to gain credence, significant benefits should accrue to the position near the cue (invalid-near) and to the position opposite the cue (valid-opposite), whereas costs should be observed for the other two positions (invalid-irrelevant). The same pattern of results could be observed, however, if subjects stochastically determined the position to which they would attend on any given trial but over trials attended to both positions. If one assumes symmetrical costs and benefits, and that both positions are attended to fairly equally, the mean RT for either cued location should be a mixture of times that are short and long relative to those for neutral cues. Thus, no processing advantage for either position should be observed. Another possibility is that a single attentional beam could be widened to include both positions. This explanation is more difficult to reject, but if one assumes that facilitation is proportional to the density of attentional resources invested, the benefits attained in this situation should be quite small relative to the benefits attained for the Central or Peripheral groups. On the other hand, if separate attentional systems are not involved, tradeoffs, as described earlier, should arise. That is, benefits should accrue initially to the position near the peripheral cue and then switch over to the position opposite the cue as the cue leads the display by longer SOAs.

\section{Method}

Subjects. Forty-two University of Kansas students, 22 males and 20 females, participated in single 40 -min sessions. Forty subjects were undergraduates who earned credit in introductory psychology or laboratory courses, and 2 subjects were psychology graduate students. The subjects ranged from 18 to 29 years of age, and they reported normal or corrected-to-normal vision.

Apparatus and Stimuli. A program running on a Zenith Data Systems Model 150 computer controlled stimulus presentation and data collection. A custom display was fashioned from a set of green, light-emitting diode (LED) elements driven by a custom electronic circuit. These included four seven-segment digits, eight triangular arrowheads, and one round fixation element.

As shown in Figure 1, the four digits formed an imaginary plus sign centered on a black, perforated circuit mounting board which was covered with gray Plexiglas to improve contrast. The round fixation element was located at the center of the imaginary plus sign and was masked with black paint so that only a pinpoint of light shone through. The center of each digit was $4.3 \mathrm{~cm}$ (about $1.68^{\circ}$ of visual angle) from the central fixation point (at a viewing distance of about $147 \mathrm{~cm}$ ). Two triangular arrowheads indicated each digit's spatial position. The arrows near the center dot served as central cues; those near the digits served as peripheral cues. Each arrowhead was $4 \mathrm{~mm}\left(.16^{\circ}\right)$ in length and $3.5 \mathrm{~mm}\left(.14^{\circ}\right)$ in width.

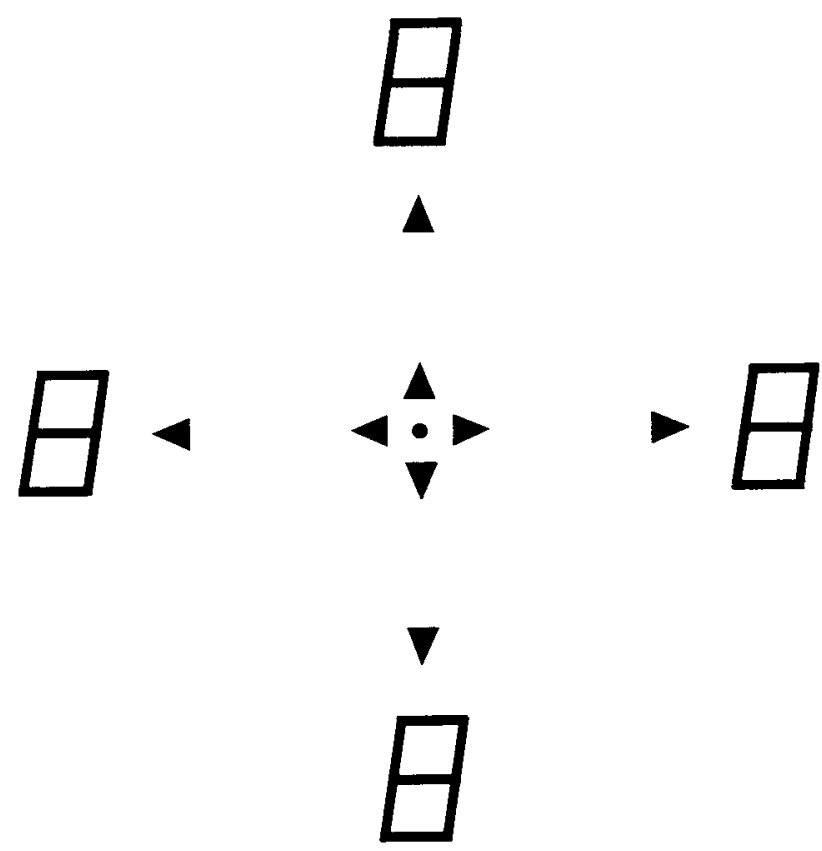

Figure 1. A representation of the display used in Experiments 1 and 2 . The layout is approximately to scale.

The central arrow's nearest edges were $4 \mathrm{~mm}\left(.16^{\circ}\right)$ from the center dot; the edges of the peripheral pointers were displaced $2.8 \mathrm{~cm}$ $\left(1.1^{\circ}\right)$ from center (horizontal axis) and $2.4 \mathrm{~cm}\left(.94^{\circ}\right)$ from center (vertical axis). The tips of the peripheral arrows were separated from the nearest edges of the digits by about $7 \mathrm{~mm}\left(.27^{\circ}\right)$.

The stimuli were five different numerals produced by illuminating the appropriate segments of a given display element. Targets were the numerals 2 and $5 ; 3,6$, and 9 served as distractors. Each numeral was $15 \mathrm{~mm}\left(.58^{\circ}\right)$ high, and $8 \mathrm{~mm}\left(.31^{\circ}\right)$ wide. These numerals were chosen because each could be formed by illuminating exactly five of the seven segments of any element.

Design. The subjects were assigned randomly to three groups of 14 each. The Central group was instructed to attend to display positions indicated by central cue arrows, and the Peripheral group was instructed to attend to the peripheral cue arrows. The remaining, Opposite group was also cued by peripheral arrows but was instructed to attend to the position diametrically opposite to the one pointed at by the arrow. Each subject received 288 experimental trials consisting of repeated measurements on the variables of SOA (with levels $0,50,100$, and $150 \mathrm{msec}$ ), and cue validity, which had three levels for the Central and Peripheral groups (valid, neutral, and invalid), and four levels for the Opposite group (validopposite, neutral, invalid-near, and invalid-irrelevant). On invalidnear trials, the cue arrow designated the diametrically opposite position in display, but the target appeared next to the arrow's tip. On invalid-irrelevant trials, the target appeared at one of the two positions along the other axis, neither at the arrow's tip nor diametrically opposite it. The targets 2 and 5 were used equally often in each cell of the design.

Procedure. After receiving preliminary instructions, each subject was allowed approximately $10 \mathrm{~min}$ to adapt to the diminished lighting of the laboratory before receiving final instructions and four example trials (half-time presentations of one valid trial, two invalid trials, and one neutral trial). Following the examples, the subject received 32 practice trials drawn randomly from the experimental trials. During the experimental trials, short breaks (typically $30 \mathrm{sec}$ or less) were allowed every 48 trials. Each subject received the experimental trials in a different random order. 
The instructions for the Central and Peripheral groups were identical. The subjects were advised to center their gazes on the fixation point and to avoid eye movements until a response had been made. They were told to press a left-hand response button for the target 2 and a right-hand button for the target 5 . The instructions emphasized both speed of response and accuracy and fully informed the subjects of the $80 \%$ validity of the cue arrow and the strategic advantage of orienting to the display position designated by it. The instructions to the Opposite group differed only in that subjects were encouraged to attend to the position opposite the arrow, because that position contained a target on $80 \%$ of the trials.

At the beginning of each trial, the fixation point was illuminated for $1 \mathrm{sec}$, followed by the illumination of one cue arrow (valid or invalid trials) or all four cue arrows (neutral trials). Only the peripheral arrows were used for the Peripheral and Opposite groups; only the central arrows were used for the Central group. ${ }^{1}$ After an SOA of $0,50,100$, or $150 \mathrm{msec}$, four different digits (one target and three distractors) appeared for $100 \mathrm{msec}$, after which the entire display (fixation point, arrow(s), and four digits) was extinguished. ${ }^{2}$ Response latency was measured from the onset of the four digits. When the subject pressed the wrong button or failed to respond within $2 \mathrm{sec}$, the computer emitted a brief, $400-\mathrm{Hz}$ tone as error feedback. A response occurring within $200 \mathrm{msec}$ was ignored, although an additional response could be recorded if the first one was too early. The experimenters noticed few, if any, double responses. Trials occurred at a fixed rate of 1 every $4 \mathrm{sec}$. Neutral trials composed $16.7 \%$ of the total trials. On the nonneutral trials, $80 \%$ of the cues were valid. On invalid trials, either target was equally likely to appear at any of the three uncued positions.

Prior to analysis, trials were discarded if the RT was more than twice the mean of any cell for each subject. (Fewer than $0.6 \%$ of the correct responses were thus eliminated.) Also discarded were instances when no response was made within $2 \mathrm{sec}$, or when an inadvertent response was made. (Fewer than $0.2 \%$ of the data fit either of these two categories.)

\section{Results}

Because of task differences and the fact that there were four levels of cue validity for the Opposite group and only three for the others, the data from the Opposite group were analyzed separately.
Central and Peripheral groups. As shown in Figure 2, RTs were shortest for validly cued trials and longest for invalidly cued trials. Costs and benefits were smallest at $0 \mathrm{msec}$, emerged rapidly by $50 \mathrm{msec}$, and were largest at $150 \mathrm{msec}$. The data were submitted to a 2 (cue type) $\times 3$ (cue validity) $\times 4$ (SOA) mixed analysis of variance (ANOVA), with repeated measures on the second and third factors. Significant main effects were found for cue validity $[F(2,52)=37.14, p<.001]$ and for SOA $[F(3,78)=3.89, p=.012]$. The cue validity $\times$ SOA interaction was also significant $[F(6,156)=20.75$, $p<.001]$. Although it was hypothesized that costs and benefits should be greater and should accrue more rapidly for peripheral cues than for central cues, negligible differences were found, and neither cue type nor its interaction with any other factor approached significance.

Costs plus benefits (mean RT for invalid trials minus that for valid trials), presented in Figure 3, also show marked similarities across cue type in both time course and magnitude. These data were submitted to a 2 (cue type) $\times 4$ (SOA) mixed ANOVA. Contrary to Jonides's evidence for greater costs plus benefits for peripheral cuing, no significant effect of cue type was observed $(F<1)$. The only significant effect observed was that of SOA $[F(3,78)=34.88, p<.001]$. Because of the a priori hypothesis that costs plus benefits should differ over SOA for the two cue types, the simple main effect of cue type at each SOA was tested. Again, no significant effects were observed.

The error data (see Table 1) were submitted to an ANOVA, using factors identical to those used for the RT data. The mean error rate of the Peripheral group (4.6\%) exceeded that of the Central group $(2.1 \%)[F(1,26)=$ $5.25, p=.03]$. Also, a main effect of cue validity was observed $[F(2,52)=4.22, p=.02]$. The error rate for invalid trials $(4.6 \%)$ exceeded that for neutral $(2.8 \%)$ and

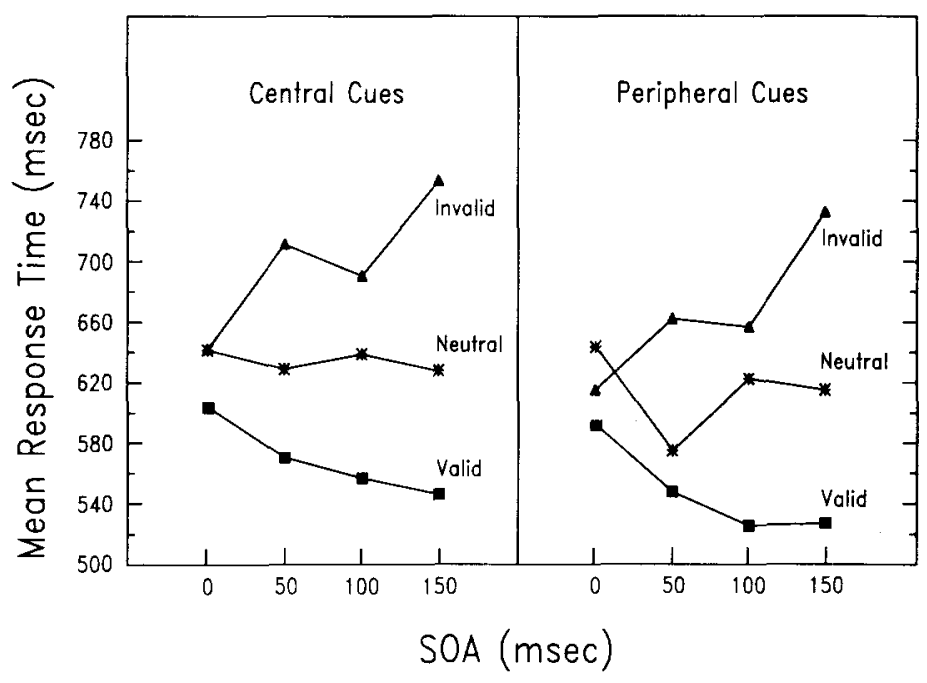

Figure 2. Central and Peripheral groups, Experiment 1: Mean response time plotted against stimulus onset asynchrony for each cue type. 


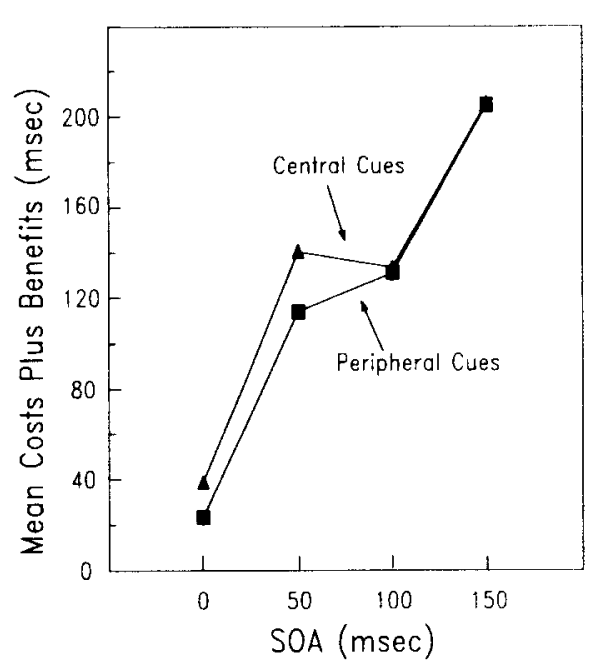

Figure 3. Central and Peripheral groups, Experiment 1: Mean costs plus benefits plotted against stimulus onset asynchrony.

valid (2.6\%) trials. Error rates were positively, but not significantly, correlated (across 24 cells) with RT $[r=$ $.31, t(22)=1.56, p=.135$ ], eliminating the possibility for extensive speed-accuracy tradeoffs.

Opposite group. The RT data, shown in Figure 4, and the error data, shown in Table 1 , were submitted to separate 4 (cue validity) $\times 4$ (SOA) ANOVAs, with repeated measures on both factors. Cue validity had a significant effect on RT $[F(3,39)=6.39, p=.001]$, and it interacted with SOA $[F(9,117)=4.02, p<.001]$. At the longer SOAs $(100$ and $150 \mathrm{msec})$, the subjects responded more rapidly to a target that appeared near the peripheral cue (invalid-near) than to one that appeared away from it (invalid-irrelevant and valid-opposite), despite instructions to attend to the opposite location. Separate analyses at each SOA with subsequent NewmanKeuls tests revealed no significant differences in RT between invalid-irrelevant and valid-opposite trials.

No significant effects were observed in the error data (see Table 1). The unweighted error rate for the Opposite group was $4.0 \%$. Error rates were positively and significantly correlated (across conditions) with RT $[r=$ $.523, t(14)=2.30, p=.037$ ]

\section{Discussion}

In Experiment 1, we found no significant differences in either the time course or the overall magnitude of costs plus benefits associated with peripheral cues as opposed to central cues. Instead, for both the Central group and the Peripheral group, costs plus benefits emerged rapidly and remained equivalent through all four SOAs. These results contrast with Jonides's (1981) findings of larger costs and benefits for peripheral cues, which he attributed to their superiority in eliciting attentional focus. The results also contrast with those of Müller and Rabbitt (1989), who observed differences in the magnitude of costs and benefits within the same range of SOAs used in Experiment 1. Caution should be exercised in trying to make direct comparisons with their data, however. The central and peripheral cues in Experiment 1 were designed to be as similar as possible, with the exception of retinal eccentricity, whereas Müller and Rabbitt compared the effects of central arrows with parafoveal flashes, thus confounding the central-peripheral distinction with other variables such as shape, size, and brightness of the cues.

One could argue, however, that in Experiment 1 we found no differences between central and peripheral cues because the peripheral cues were not "peripheral enough." That is, the peripheral cues might have been too close to the fovea to activate automatic attentional capture. Support for the adequacy of the peripheral cues used in Experiment 1 comes from the test of another hypothesis, derived from Jonides (1981), which states that attention is disengaged from an ongoing peripheral orientation only with difficulty. This hypothesis is supported by the data from the Opposite group (see Figure 4). In the Opposite condition, the cue served both as a high-validity volitional cue (valid-opposite trials) and as a low-validity peripheral cue (invalid-near trials). The pattern of costs and benefits for the opposite cue does not resemble the pattern observed when the peripheral cue has literal spatial significance (see the right panel of Figure 2). Instead, a target digit is detected most rapidly when it occupies the position near the cue arrow rather than the opposite position, where attention was supposed to be directed and where the target most typically appeared. Clearly, the large costs and benefits observed in Figure 2 do not appear in Figure 4. Because the subjects had little to gain from intentionally attending to the character near the cue arrow (where the target digit occurred only $6.7 \%$ of the time), one could argue that the peripheral cue captured their attention. Even by the longest SOA, the subjects showed little ability to disengage attention from the character near the cue arrow and reallocate it to the opposite position in the display.

Table 1

Error Rates (\%) for the Central, Peripheral, and Opposite Groups of Experiment 1

\begin{tabular}{lcccc} 
& \multicolumn{5}{c}{ SOA } \\
\cline { 2 - 5 } Cue Validity & 0 & 50 & 100 & 150 \\
\hline & Central Group & & \\
Valid & 1.7 & 3.1 & 1.2 & 1.2 \\
Neutral & 1.2 & 2.4 & 0.6 & 1.8 \\
Invalid & 5.1 & 1.9 & 1.3 & 3.9 \\
& & & & \\
& Peripheral Group & & \\
Valid & 3.2 & 3.0 & 3.1 & 4.4 \\
Neutral & 6.0 & 3.6 & 3.0 & 3.6 \\
Invalid & 3.6 & 5.8 & 7.1 & 8.9 \\
& & & & \\
& Opposite & Group & & \\
Valid-Opposite & 3.6 & 4.1 & 3.6 & 3.9 \\
Neutral & 4.2 & 4.8 & 4.8 & 3.6 \\
Invalid-Irrelevant & 0.9 & 7.2 & 9.4 & 5.4 \\
Invalid-Near & 1.8 & 1.4 & 1.4 & 3.6 \\
\hline
\end{tabular}

Note-SOA $=$ stimulus onset asynchrony (in milliseconds). 


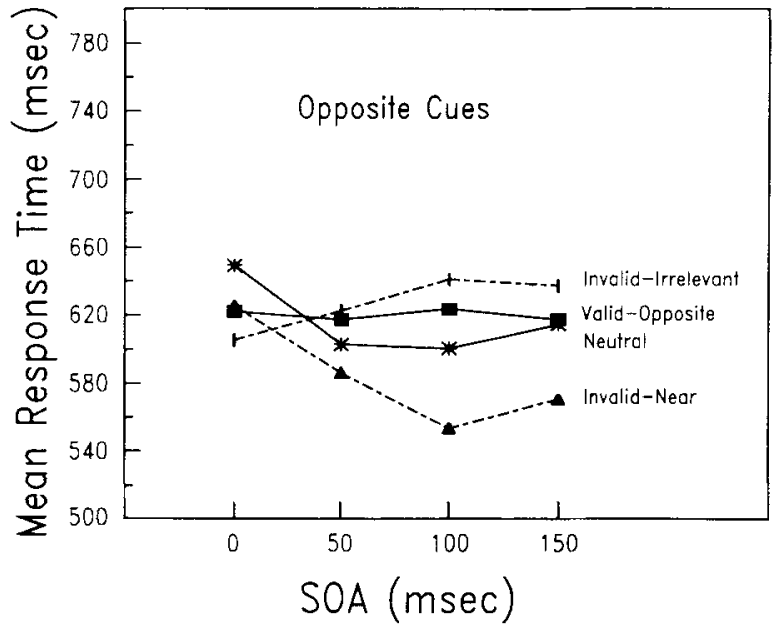

Figure 4. Opposite group, Experiment 1: Mean response time plotted against stimulus onset asynchrony for each cue type. The peripheral arrow served as both a low-validity nonvolitional cue and a high-validity volitional cue. The invalid-near curve indicates trials on which the critical digit appeared next to the peripheral cue. The valid-opposite curve indicates trials on which the critical digit appeared diametrically opposite the peripheral cue. The invalid-irrelevant curve indicates trials on which the critical digit appeared neither near nor opposite the cue arrow.

An alternative to the automatic capture hypothesis is that the subjects first had to engage the cue with the attentional spotlight in order to interpret it. Our subjective impression, however, was that the arrow appeared as a flash of light in a particular position relative to the fixation point, and that this positional information was sufficient to trigger an attentional response to the appropriate location. The fact that the arrow actually pointed in a particular direction was of secondary importance, inasmuch as neutral-pointing spots or bars could have served the same purpose.

The hypothesis that volitional and nonvolitional cues elicit separate attentional systems is not supported by the present data. If the separable systems hypothesis were true, significant benefits should have been found both for the position next to the cue (invalid-near) and for the position diametrically opposite the cue (valid-opposite). In the present study, benefits were observed only for the position near the cue (invalid-near). Conceivably, effects might have been observed at both positions had the maximum cue-target SOA been longer; however, large costs and benefits were observed for the Central group (volitional cues) within the same time course. The difficulty does not seem to lie in the time needed to attend volitionally to the opposite position but in the time needed to disengage attention from the region indicated by the peripheral cue.

\section{EXPERIMENT 2}

It is possible that lack of practice, not lack of time, might explain why subjects in the Opposite condition of Experiment 1 were apparently unable to disengage and refocus attention as predicted. In Experiment 2, we tested whether, with extended practice, subjects might learn to circumvent an apparently automatic attentional process and become able to refocus attention rapidly and volitionally. In this experiment, subjects participated in several sessions that were essentially replications of the Opposite condition of Experiment 1.

\section{Method}

Subjects. Four male subjects served in from 8 to 10 sessions. Two were undergraduates participating in exchange for research credits, and 2 were coauthors of the present paper (J.F.J. and H.K.). One of the 2 undergraduates had served in the Opposite group of Experiment 1, and his data from the previous experiment are included in the second experiment as his first session.

Design and Procedure. The conditions and procedure were the same as for the Opposite group of Experiment 1, except that each session of the present experiment consisted of 576 trials rather than 288 trials. Also, no example trials were used to start the sessions. Rather, 32 trials were chosen randomly from the pool of 576 to serve as practice. Finally, all subjects participated in at least eight sessions until an asymptotic performance level was reached. In general, sessions were run on consecutive days, but some longer breaks of 2-3 days occurred occasionally.

Three levels of practice (low, medium, and high) were determined by visual examination of the RT data. On the basis of similarity of performance, sessions were combined to form the three levels: low (Sessions 1-2), medium (Sessions 3-5), and high (Sessions 6-8 for all subjects, plus Session 9 for 2 subjects, and Session 10 for 1 subject).

\section{Results}

Overall, $3.5 \%$ of the trials (using a weighted average) resulted in response errors. The error data (see Table 2 ) were submitted to a 4 (cue validity) $\times 4$ (SOA) $\times 3$ (practice) analysis with repeated measures on all factors. Only the main effect for SOA attained significance $[F(3,9)=$ $4.25, p=.04]$. The mean error rates were $3.0 \%, 3.6 \%$, $2.8 \%$, and $4.0 \%$, respectively, for $0-, 50-, 100-$, and $150-$ msec SOAs.

Table 2

Error rates (\%) for Experiment 2

\begin{tabular}{|c|c|c|c|c|}
\hline \multirow[b]{2}{*}{ Cue Validity } & \multicolumn{4}{|c|}{ SOA } \\
\hline & 0 & 50 & 100 & 150 \\
\hline \multicolumn{5}{|c|}{ Low Practice } \\
\hline Valid-Opposite & 3.0 & 2.6 & 3.5 & 3.8 \\
\hline Neutral & 2.6 & 1.6 & 2.1 & 1.6 \\
\hline Invalid-Irrelevant & 3.1 & 3.9 & 2.3 & 4.7 \\
\hline Invalid-Near & 1.6 & 4.7 & 1.6 & 3.1 \\
\hline \multicolumn{5}{|c|}{ Medium Practice } \\
\hline Valid-Opposite & 3.2 & 4.0 & 3.7 & 3.7 \\
\hline Neutral & 2.4 & 3.5 & 1.4 & 3.8 \\
\hline Invalid-Irrelevant & 3.1 & 2.1 & 3.1 & 5.8 \\
\hline Invalid-Near & 4.2 & 4.2 & 3.1 & 6.3 \\
\hline \multicolumn{5}{|c|}{ High Practice } \\
\hline Valid-Opposite & 3.5 & 3.7 & 4.7 & 4.0 \\
\hline Neutral & 3.3 & 4.2 & 2.0 & 2.2 \\
\hline Invalid-Irrelevant & 3.4 & 5.0 & 4.2 & 6.7 \\
\hline Invalid-Near & 3.1 & 3.5 & 2.0 & 2.4 \\
\hline
\end{tabular}

Note-SOA $=$ stimulus onset asynchrony (in milliseconds). 
Fewer than $0.15 \%$ of the correct responses were counted as outliers in the RT data and removed. The RT data shown in Figure 5 were analyzed in a 4 (cue validity) $\times 4$ (SOA) $\times 3$ (practice) repeated-measures analysis. ${ }^{3}$ There was a significant main effect for cue validity $[F(3,9)=$ $7.00, p=.01]$ and a marginally significant main effect for practice $[F(2,6)=5.07, p=.051]$. Mean RT for invalid-irrelevant trials $(505 \mathrm{msec})$ was longer than that for invalid-near $(477 \mathrm{msec})$ and neutral $(478 \mathrm{msec})$ trials, and mean RT was shortest for valid-opposite trials (460 msec). Mean RT declined from $506 \mathrm{msec}$ with low levels of practice to 470 and $465 \mathrm{msec}$ with moderate and high levels, respectively. These outcomes must be qualified by a marginally significant cue validity $\times$ practice interaction $[F(6,18)=2.60, p=.054]$ and a significant validity $\times$ SOA interaction $[F(9,27)=7.43, p<.001]$. Costs and benefits increased across SOA, as shown in Figure 5. Figure 6 shows the validity $\times$ practice interaction collapsed across SOA. The most notable change in mean RT with practice occurs for invalid-near trials. During the first two sessions, on the average, benefits were observed for responses to invalidly cued targets near the cue arrow, but only costs were observed in the latter sessions.

\section{Discussion}

The change with practice from benefit to cost for invalidly cued targets near the cue arrow suggests a fundamental alteration in subjects' attentional responses. Initially, attention is captured by the onset of the peripheral cue, a process that is difficult to suppress once initiated. With practice, however, subjects are able to disengage and rapidly remobilize attention to the position diametri- cally opposite the cue arrow. Benefits diminish with practice at the invalid-near position, and they increase with practice at the valid-opposite location. The initially strong attentional response to the spatial channel containing the onset of the cue is, through practice, modified until cost is observed for identifying the targets near the cue and benefit is observed for identifying targets diametrically opposite the cue in the display, as was predicted for the operation of a single attentional system.

Subjects do not merely inhibit responses to invalidly cued targets near the cue arrow because, with high practice, RTs for invalid-near trials are no longer than those for invalid-irrelevant trials (see right panel of Figure 5). With low practice (left panel), the invalid-near curve generally falls below the neutral curve. The benefit is quite noticeable at 50-msec SOA, as would be expected if attention were captured by the onset of the peripheral cue. The benefit diminishes at longer SOAs, as would be expected if attention were volitionally disengaged from the initial capture. (Benefit at $0-\mathrm{msec}$ SOA is probably an artifact of the elevated neutral point, which might be explained by supposing that the onset of all four arrows in the neutral-cue condition resulted in some disruption of processing or masking of the characters.)

In the Opposite group of Experiment 1 (see Figure 4), the only observable effect was that of benefit for the invalid-near position. In the present experiment, however, strong costs were observed for the invalid-irrelevant positions, and, even with low practice, a benefit was observed for the valid-opposite position at $150-\mathrm{msec}$ SOA. Benefits were greatest at 100 - and 150 -msec SOAs for the invalid-near position in Experiment 1 (see Figure 4), but had diminished at 100- and 150-msec SOAs for low

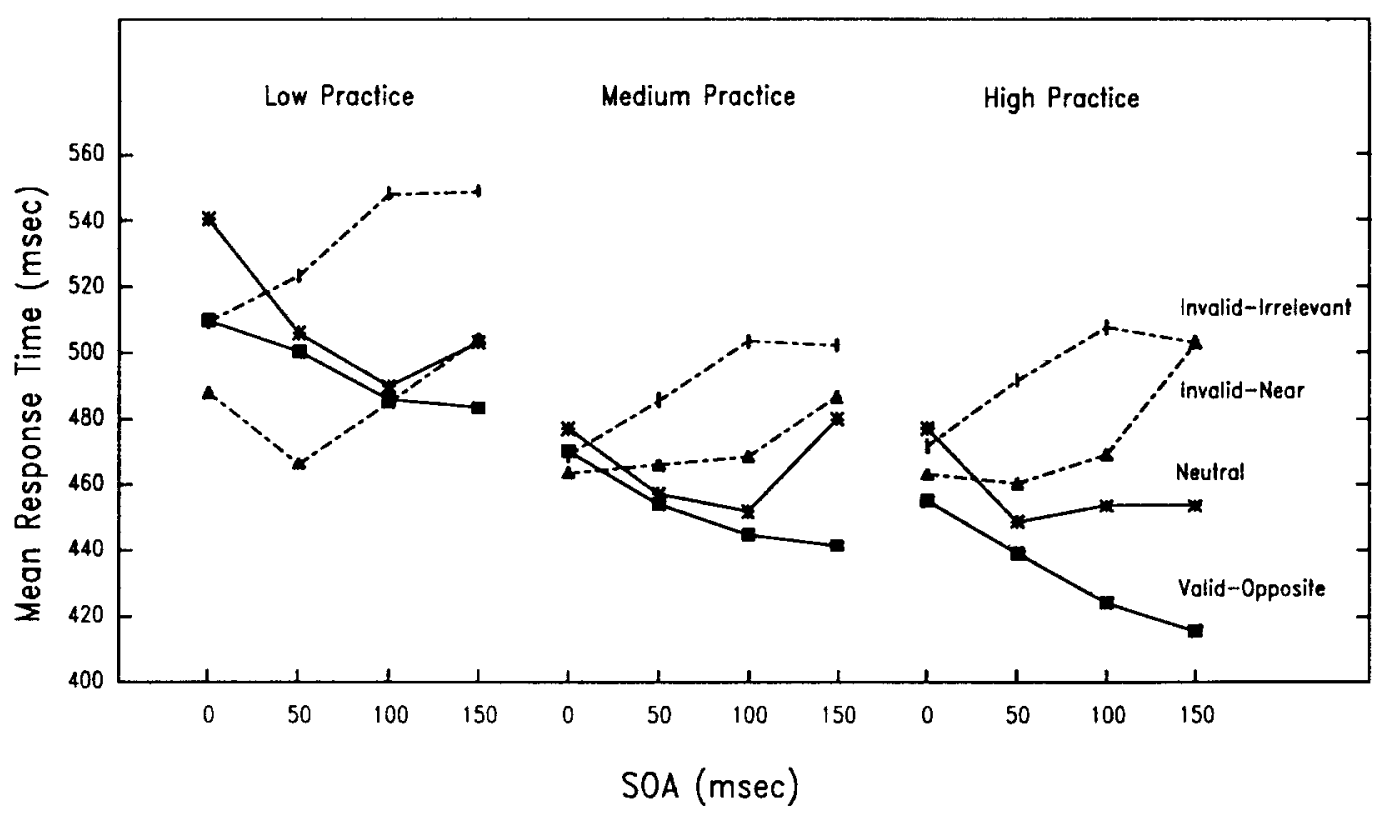

Figure 5. The mean response time data of Experiment 2, plotting cue validity against stimulus onset asynchrony for each of the three levels of practice. The first sessions of practice are represented in the left panel, sessions three to five in the middle panel, and six to eight, nine, or ten, in the right panel. 


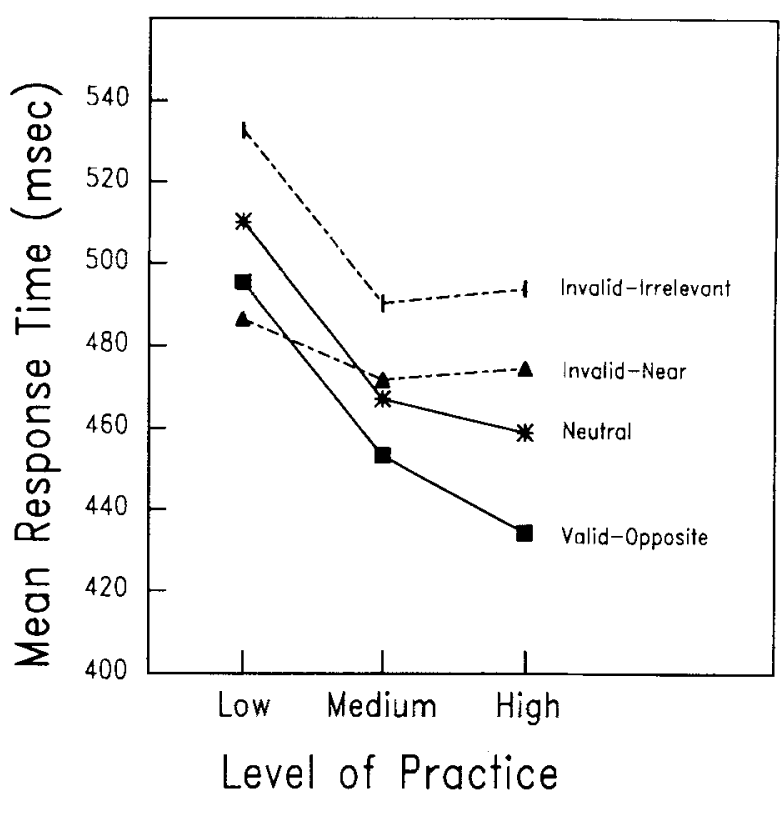

Figure 6. The mean response time data of Experiment 2 collapsed across SOA.

practice sessions in Experiment 2 (see Figure 5). The resolution of the apparent incongruity is that about four times as much practice is represented in the first panel of Figure 5 than is in Figure 4. With two sessions of practice, attention is captured by the peripheral cue at $50-\mathrm{msec}$ SOA, but at longer SOAs subjects are beginning to withdraw attention from near the cue arrow and to refocus it on the diametrically opposite position. With further practice, as shown in the middle and right panels of Figure 5, benefit is realized at progressively shorter SOAs for valid-opposite trials, and cost is observed to develop for invalid-near trials at the two longest SOAs.

Schneider and Shiffrin (1977) and Shiffrin and Schneider (1977) have demonstrated that through extensive training with consistent stimulus-response mapping, automatic attentional responses can develop to specific stimuli or classes of stimuli. The crucial difference between their studies and ours is that their subjects developed automatic attentional responses to stimuli that did not previously elicit such responses, whereas our subjects gained volitional control of a presumably automatic attentional response. The subjects in Experiment 2 became progressively faster at target identification because they rapidly regained control over attention, not because they developed automatic attentional responses to targets 2 and 5. If targets had been detected automatically, then no costs would have been observed when they appeared in invalidly cued positions. ${ }^{4}$

In previous demonstrations of attentional capture by abrupt-onset stimuli (e.g., Yantis \& Jonides, 1984), subjects have had no prior or competing intentions to focus attention elsewhere. If attentional responses to abruptonset stimuli can be suppressed given competing intentions (a seemingly necessary and reasonable assumption), they fail one of the primary criteria for automaticity, that of intentionality (resistance to suppression). The ability of our subjects to gain volitional control of attentional focus argues against the automatic nature of attentional capture by abrupt-onset stimuli. At best, attentional capture by abrupt onset is only partially automatic (see Kahneman \& Treisman, 1984). Yantis and Jonides (1990) propose a model in which an abrupt onset is always marked as a high-priority event, but if attention is already focused elsewhere, the "interrupt" initiated by the onset is placed in a queue until it can be serviced. Whether an onset stimulus will capture attention may depend in part on whether competing intentions have been established. In turn, the task context will doubtlessly affect how easily prior or competing intentions can be established.

\section{GENERAL DISCUSSION}

In Experiment 1, peripheral cues did not generate greater costs and benefits than did central cues, and their attentional effects appeared no sooner than those of central cues. Both of these results, however, are at odds with those of Müller and Rabbitt (1989, Experiment 1), who found that the effects of peripheral cues were stronger and appeared earlier than those of central cues. Perhaps their confounding of the central-peripheral distinction with other cue factors led to an overestimate of the magnitude and rapidity of attentional capture by an extrafoveal event. The similarity of the attentional responses generated by central and peripheral cues in the present study provides no evidence for assuming that two separate attention control mechanisms exist.

One possible limitation of the peripheral cues used in Experiment 1 is that they simply were not peripheral enough to stimulate strongly the transient detectors that presumably are responsible for eliciting automatic attentional responses. The results from the Opposite group of Experiment 1 belie that explanation, however. Attentional capture by the peripheral cue occurred, despite instructions to attend opposite the cue. Only with considerable training were subjects able to circumvent attentional capture (Experiment 2). A counterargument would be that attention always came under volitional control, but that insufficient time was allowed for its redirection. Such time limitation would explain the failure to observe costs for the invalid-near and benefits for the valid-opposite positions of Experiment 1 and the early stages of Experiment 2. The effect of extended practice in Experiment 2, then, was to shorten the time required for redirection, presumably by automating the process.

In Experiment 1, no evidence was found for separate automatic and volitional attentional systems that could be simultaneously directed to spatially disparate locations. Apparently, visual selective attention depends on a single system with at least two primary sources of control over attentional focus. One source is volitional and the other is partially automatic, triggered by visual channels that detect transients such as abrupt onset, motion, or flicker (e.g., Yantis \& Jonides, 1984). 
The results of Experiment 2 demonstrate that attentional capture by a single abrupt-onset cue is neither inevitable nor unalterable. Intuitively, one might have expected attentional capture to have been more easily overridden than was apparent in the present experiments. However, the task demands of attending to the position opposite the cue arrow were especially difficult, because subjects could neither establish prior intention nor ignore the cue itself, if benefits in performance were to be obtained. Yantis and Jonides (1990) have shown that the strength of capture by an abrupt-onset stimulus can be modified by prior focus of attention elsewhere. The present study extended this modification to include voluntary redirection of attention away from the onset cue itself. The susceptibility of attentional capture to correction by volition suggests that the attentional response to a sudden onset is at best only partially automatic, since it can be circumvented by the establishment of a competing perceptual expectancy. Even if this tendency is not realized until the location of the abrupt onset is identified, the apparently automatic capture of attention can be brought under control with practice, such that processing benefits for a disparate location can be obtained within $100 \mathrm{msec}$ of the onset of the cue.

\section{REFERENCES}

Briand, K. A., \& Klein, R. M. (1987). Is Posner's "beam" the same as Treisman's "glue"'?: On the relation between visual orienting and feature integration theory. Journal of Experimental Psychology: $\mathrm{Hu}$ man Perception \& Performance, 13, 228-241.

ERiksen, C. W., \& Collins, J. F. (1969). Temporal course of selective attention. Journal of Experimental Psychology, 80, 254-261.

ERIKSEN, C. W., \& ST. James, J. D. (1986). Visual attention within and around the field of focal attention: A zoom lens model. Perception \& Psychophysics, 40, 225-240.

ERIKSEN, C. W., \& YEH, Y. (1985). Allocation of attention in the visual field. Journal of Experimental Psychology: Human Perception \& Performance, 11, 583-597.

Fuchs, A. F. (1971). The saccadic system. In P. Bach-y-Rita, C. C. Collins, \& J. E. Hyde (Eds.), The control of eye movements (pp. 343362). New York: Academic Press.

JoNiDES, J. (1981). Voluntary versus automatic control over the mind's eye movement. In J. B. Long \& A. D. Baddeley (Eds.), Attention and performance $I X$ (pp. 187-203). Hillsdale, NJ: Erlbaum.

JONIDES, J., \& YANTIS, S. (1988). Uniqueness of abrupt visual onset in capturing attention. Perception \& Psychophysics, 43, 346-354.

Kahneman, D., \& Treisman, A. (1984). Changing views of attention and automaticity. In R. Parasuraman \& D. R. Davies (Eds.), Varieties of attention (pp. 29-57). New York: Academic Press.

LABERGE, D. (1981). Automatic information processing: A review. In J. B. Long \& A. D. Baddeley (Eds.), Attention and performance IX (pp. 173-186). Hillsdale, NJ: Erlbaum.

LABERGE, D. (1983). Spatial extent of attention to letters and words. Journal of Experimental Psychology: Human Perception \& Performance, 9, 371-379.

Mayfrank, L., Kimmig, H., \& Fischer, B. (1987). The role of attention in the preparation of visually guided saccadic eye movements in man. In J. K. O'Regan \& A. Levy-Schoen (Eds.), Eye movements: From physiology to cognition (pp. 37-45). New York: Elsevier/NorthHolland.

Müller, H. J., \& RabBitT, P. M. A. (1989). Reflexive and voluntary orienting of visual attention: Time course of activation and resistance to interruption. Journal of Experimental Psychology: Human Perception \& Performance, 15, 315-330.

Posner, M. I. (1980). Orienting of attention. Quarterly Journal of Experimental Psychology, 32, 3-25.

PosNer, M. I., COHEN, Y. (1984). Components of visual orienting. In H. Bouma \& D. G. Bouwhuis (Eds.), Attention and performance $X$ (pp. 531-556). Hillsdale, NJ: Erlbaum.

Russo, E. J. (1978). Adaptation of cognitive processes to the eye movement system. In J. W. Senders, D. F. Fischer, \& R. A. Monty (Eds.), Eye movements and the higher psychological functions (pp. 89-112). Hillsdale, NJ: Erlbaum.

SCHNEIDER, W., \& SHIFFrin, R. M. (1977). Controlled and automatic human information processing: I. Detection, search, and attention. Psychological Review, 84, 1-66.

ShIfFrin, R. M. (1988). Attention. In R. C. Atkinson, R. J. Hermstein, G. Lindzey, \& R. D. Luce (Eds.), Stevens' Handbook of experimental psychology: Vol. 2. Learning and cognition (2nd ed., pp. 739-811). New York: Wiley.

SHIFFrIN, R. M., SCHNEIDER, W. (1977). Controlled and automatic human information processing: II. Perceptual learning, automatic attending, and a general theory. Psychological Review, 84, 127-190.

TODD, J. T., \& VAN GELDER, P. (1979). Implications of a transientsustained dichotomy for the measurement of human performance. Journal of Experimental Psychology: Human Perception \& Performance, $5,625-638$.

Treisman, A. M. \& Gelade, G. (1980). A feature integration theory of attention. Cognitive Psychology, 12, 97-136.

YanTIS, S., \& Jonides, J. (1984). Abrupt visual onsets and selective attention: Evidence from visual search. Journal of Experimental Psychology: Human Perception \& Performance, 10, 601-621.

YANTIS, S., \& JonidES, J. (1990). Abrupt visual onsets and selective attention: Voluntary versus automatic allocation. Journal of Experimental Psychology: Human Perception \& Performance, 5, 121-134.

\section{NOTES}

1. Four pilot subjects were run in both a simple and a choice RT task using only the arrows as stimuli. In both tasks, RT was slightly but nonsignificantly shorter for the peripheral arrows. Thus, any attentional differences observed in Experiment 1 between the Central and Peripheral groups are unlikely to be due to perceptual differences between central and peripheral arrows.

2. SOAs were capped at $150 \mathrm{msec}$ to avoid the potential confounding of eye movements and attentional effects. With the stimuli present for $100 \mathrm{msec}$ at the longest SOA, an eye movement would have to be completed in under $250 \mathrm{msec}$ in order to fixate a potential target before it disappeared. With a fixation point present, the average saccade latency is about $250 \mathrm{msec}$, with few saccades occurring earlier than 200 msec (e.g., Mayfrank, Kimmig, \& Fischer, 1987). This compares reasonably with other consensus estimates of the latency to initiate small eye movements (e.g., Fuchs, 1971; Russo, 1978).

3. A special ANOVA also was performed which included subject type (coauthor vs. naive) as a factor. Neither subject type nor its interaction with any other factor attained significance $(p>.05)$.

4. Why did an automatic attention response to the presence of the targets not develop? After all, the targets were consistently mapped to the responses. Shiffrin and Schneider (1977) found that even with consistently mapped training an automatic attentional response can develop only if there are no competing demands on attention. In Experiment 2 there were at least two competing demands on attention: (1) the automatic capture exhibited by the peripheral cue itself, and (2) the task requirement to attend to the position opposite the cue arrow. Also, consistent training may not lead to automatic detection when target-distractor confusability is high (Shiffrin, 1988). The seven-segment characters used in Experiment 2 permitted little featural diversity among targets and distractors, since neither obliques nor curves, for instance, could be represented.

(Manuscript received June 5, 1989 . revision accepted for publication April 2, 1990.) 